TRANSACTIONS OF THE

AMERICAN MATHEMATICAL SOCIETY

Volume 357, Number 10, Pages 3953-3969

S 0002-9947(05)04010-9

Article electronically published on May 20, 2005

\title{
TURING PATTERNS IN THE LENGYEL-EPSTEIN SYSTEM FOR THE CIMA REACTION
}

\author{
WEI-MING NI AND MOXUN TANG
}

\begin{abstract}
The first experimental evidence of the Turing pattern was observed by De Kepper and her associates (1990) on the CIMA reaction in an open unstirred gel reactor, almost 40 years after Turing's prediction. Lengyel and Epstein characterized this famous experiment using a system of reactiondiffusion equations. In this paper we report some fundamental analytic properties of the Lengyel-Epstein system. Our result also indicates that if either of the initial concentrations of the reactants, the size of the reactor, or the effective diffusion rate, are not large enough, then the system does not admit nonconstant steady states. A priori estimates are fundamental to our approach for this nonexistence result. The degree theory was combined with the a priori estimates to derive existence of nonconstant steady states.
\end{abstract}

\section{INTRODUCTION}

The regeneration phenomenon of hydra, discovered by A. Trembley in 1744 [ $\mathrm{Tr}$, is among the earliest and most well-known examples in morphogenesis. Attempting to model this interesting and important phenomenon in biological pattern formation, A. Turing proposed the striking idea of "diffusion-driven instability" in 1952. More precisely, in [T] Turing argued, in a system of two interactive substances, different diffusion rates could lead to nonhomogeneous distributions of such reactants. Although this idea was later successfully developed, on the theoretical side, by A. Gierer and H. Meinhardt [GM] in modeling the regeneration phenomenon of hydra, and was further verified mathematically to be capable of producing spikes and peaks (see e.g. $[\mathrm{N}]$ ), Turing's revolutionary idea has never been experimentally verified in hydra to this date.

The first experimental evidence of the Turing pattern was observed in 1990 by De Kepper et al. ([CDBD] and [DCDB] $)$ on the chlorite-iodide-malonic acid and starch reaction (CIMA reaction) in an open unstirred gel reactor, almost 40 years after the publication of Turing's paper. The fact that there are five reactants involved in the CIMA reaction makes the mathematical description very complicated. However, observing that three of the five reactants remain nearly constants in the CIMA reaction, Lengyel and Epstein [LE1], [LE2] were able to reduce it to a $2 \times 2$ system.

Let $\Omega$ be a bounded domain in $R^{n}$, with a smooth boundary $\partial \Omega$. Let $u=u(x, t)$ and $v=v(x, t)$ denote the chemical concentrations of the activator iodide $\left(\mathrm{I}^{-}\right)$and the inhibitor chlorite $\left(\mathrm{ClO}_{2}^{-}\right)$, respectively, at time $t>0$ and a point $x \in \Omega$. The

Received by the editors June 16, 2003.

2000 Mathematics Subject Classification. Primary 35K50, 35K57, 92D25.

(C)2005 American Mathematical Society 
Lengyel and Epstein model takes the form

$$
\begin{aligned}
& \frac{\partial u}{\partial t}=\Delta u+a-u-\frac{4 u v}{1+u^{2}} \\
& \frac{\partial v}{\partial t}=\sigma\left[c \Delta v+b\left(u-\frac{u v}{1+u^{2}}\right)\right]
\end{aligned}
$$

where $\Delta=\sum_{i=1}^{n} \frac{\partial^{2}}{\partial x_{i}^{2}}$ is the Laplace operator, carrying the spatial dependence of the reaction; $a$ and $b$ are parameters related to the feed concentrations; $c$ is the ratio of the diffusion coefficients; $\sigma>1$ is a rescaling parameter depending on the concentration of the starch, enlarging the effective diffusion ratio to $\sigma c$. In laboratory conditions, a sample of parameters is taken in the range $0<a<35$, $0<b<8, c=1.5$ and $\sigma=8$. We shall assume accordingly that all constants $a, b, c$, and $\sigma$ are positive.

We consider positive solutions to the model subject to the initial condition

$$
u(x, 0)=u_{0}(x)>0, v(x, 0)=v_{0}(x)>0, x \in \Omega,
$$

where $u_{0}, v_{0} \in C^{2}(\Omega) \cap C^{0}(\bar{\Omega})$, and the Neumann boundary condition

$$
\partial u / \partial \nu=\partial v / \partial \nu=0, x \in \partial \Omega, t>0,
$$

where $\nu$ is the unit outer normal to $\partial \Omega$.

Although various important experimental and numerical studies on the LengyelEpstein system have been conducted in the last decade (see, e.g., CK, JMBD, JS, WS]), the mathematical progress on the analytic aspects of the system has been very limited. The main purpose of this paper is to report some of the fundamental properties of this system in our preliminary studies. Our main results include both existence and non-existence for the steady states of the system (1.1)-(1.4). In Section 4, Theorems 1, 2 and 3 show that, roughly speaking, if the parameter a (related to the feed concentrations), the size of the reactor $\Omega$ (reflected by its first eigenvalue), or the "effective" diffusion rate $d=c / b$ is not large enough, then the system (1.1)-(1.4) has no nonconstant steady states. On the other hand, Theorem 4 in Section 6 guarantees that, if the parameter a lies in a suitable range, then (1.1) -(1.4) possesses nonconstant steady states for large "effective" diffusion rate $d$. These results further verify the original idea in "diffusion-driven instability" of Turing.

A priori estimates for solutions of (1.1)-(1.4) are fundamental to our approach for the nonexistence results. For the existence, we combine our a priori bounds with the degree theory. The drawback in the degree-theoretical approach is that we are not able to say much about the shape of the solution obtained this way. In [JNT we made a better description for the structure of the set of the non-constant steady states on the one-dimensional case.

\section{INVARIANT REGION}

In this section, we will show that the parabolic system (1.1)-(1.4) has an invariant region

$$
\Re_{a}=(0, a) \times\left(0,1+a^{2}\right)
$$

in the phase plane which actually attracts all solutions of this system, regardless of the initial values $u_{0}$ and $v_{0}$. 
To begin with, we first show that the initial-boundary value problem (1.1)-(1.4) has a unique solution $(u(x, t), v(x, t))$ that is defined for all $t>0$ and is bounded by some positive constants depending on $a, u_{0}$ and $v_{0}$.

Proposition 2.1. The initial-boundary value problem 1.1-1.4 admits a unique solution $(u, v)$, defined for all $x \in \Omega$ and $t>0$. Moreover, there exist two positive constants $C_{1}, C_{2}$, depending only on $a, u_{0}$ and $v_{0}$ such that

$$
C_{1}<u(x, t), v(x, t)<C_{2}, \quad x \in \Omega, t>0 .
$$

Proof. The local existence and uniqueness of solutions to the initial-boundary value problem (1.1) - (1.4) are classical; see [F]. For the global existence and the boundedness, we apply the theory of invariant region as was developed in [W]. In particular, if there exists a region

$$
\Re=\left(U_{1}, U_{2}\right) \times\left(V_{1}, V_{2}\right)
$$

in the $(u, v)$ phase plane such that the vector field

$$
\left(a-u-4 u v /\left(1+u^{2}\right), \sigma b\left(u-u v /\left(1+u^{2}\right)\right)\right)
$$

points inward on the boundary of $\Re$, then $\Re$ is a (positively) invariant rectangle, and the solution $(u, v)$ of (1.1) - (1.4) exists for all $x \in \Omega, t \geq 0$, and stays in $\Re$.

To complete the proof, we choose

$$
U_{2}=\max \left\{a, \max _{x \in \bar{\Omega}} u_{0}(x)\right\}, \quad V_{2}=\max \left\{2+U_{2}^{2}, \max _{x \in \bar{\Omega}} v_{0}(x)\right\},
$$

and

$$
U_{1}=\min \left\{a /\left(1+4 V_{2}\right), \min _{x \in \bar{\Omega}} u_{0}(x)\right\}, \quad V_{1}=\min \left\{1 / 2, \min _{x \in \bar{\Omega}} v_{0}(x)\right\} .
$$

Clearly, the initial functions $u_{0}(x)$ and $v_{0}(x)$ are enclosed by the rectangle. It is easy to verify that the vector field points into the rectangle $\Re$ along its boundary: Indeed, on the left side $u=U_{1}, V_{1} \leq v \leq V_{2}$, we have

$$
a-u-4 u v /\left(1+u^{2}\right)>a-U_{1}-4 U_{1} V_{2} \geq 0
$$

since $U_{1} \leq a /\left(1+4 V_{2}\right)$, so the vector field crosses this line in the direction pointing inside $\Re$. For the other three sides, the simple verification can be done by similar calculations. Therefore the region $\Re$ is positively invariant with respect to the vector field. We thus obtain the global existence and the uniqueness of the solutions.

The two constants $C_{1}$ and $C_{2}$ can now be chosen, respectively, by

$$
C_{1}=\min \left\{U_{1}, V_{1}\right\}>0, \quad C_{2}=\max \left\{U_{2}, V_{2}\right\}>0,
$$

which depend on $a, u_{0}$ and $v_{0}$.

We remark that the invariant region $\Re$ constructed above can be arbitrarily large because of its dependence on the initial values $u_{0}$ and $v_{0}$. However, the set $\Re_{a}$ as mentioned at the beginning of this section depends only on $a$, and it attracts all solutions of (1.1)-(1.4). More precisely, let $u=u(x, t)$ and $v=v(x, t)$ be solutions of (1.1)- (1.4). Then there is some $T \geq 0$, which may depend on $u_{0}$ and $v_{0}$, such that $(u, v) \in \Re_{a}$ for all $t>T$ and $x \in \bar{\Omega}$.

Proposition 2.2. Let $u=u(x, t)$ and $v=v(x, t)$ be the unique solutions of (1.1)(1.4). Then we have

$$
\limsup _{t \rightarrow \infty} u<a \quad \text { and } \quad \limsup _{t \rightarrow \infty} v<1+a^{2}
$$

for all $x \in \bar{\Omega}$. 
Proof. By Proposition 2.1 there exists an $\epsilon>0$ such that

$$
\epsilon<\frac{4 u v}{1+u^{2}}
$$

for all $x \in \bar{\Omega}$ and $t>0$. We put $\tilde{a}=a-\epsilon / 2$ and let $\tilde{u}=\tilde{u}(t)$ be the unique solution to the initial value problem of the ordinary differential equation

$$
\frac{d \tilde{u}}{d t}=\tilde{a}-\tilde{u}, \quad \tilde{u}(0)=2 \max _{x \in \bar{\Omega}} u_{0}(x) .
$$

Let $\hat{u}=u-\tilde{u}$. By (1.1) and (2.1) we have

$$
-\hat{u}_{t}+\Delta \hat{u}-\hat{u}=\frac{4 u v}{1+u^{2}}+\tilde{a}-a>0
$$

and $\hat{u}(x, 0)<0$. Then using the classical maximum principle for parabolic equations, combined with the Neumann boundary condition (1.4), we find that $\hat{u}(x, t)<$ 0 and so $u(x, t)<\tilde{u}(t)$ for all $t>0$ and $x \in \bar{\Omega}$.

We next derive a comparison result for $v=v(x, t)$ and a function $\tilde{v}=\tilde{v}(t)$ defined below. We remark that the maximum principle for parabolic equations does not seem to be directly applicable to this case, so we shall give an elementary argument using Hopf's boundary lemma for elliptic equations.

Let $C_{1}$ be the constant given in Proposition 2.1. Define

$$
\tilde{g}(\tilde{u}, \tilde{v})=\sup _{C_{1}<\xi<\tilde{u}}\left(\xi-\frac{\xi\left(\tilde{v}-\epsilon_{0}\right)}{1+\xi^{2}}\right),
$$

where $\epsilon_{0}>0$ and

$$
\tilde{a}^{2}+\epsilon_{0}<a^{2} .
$$

Then the function $\tilde{v}(t)$ is defined to be the unique solution to the initial value problem

$$
\frac{d \tilde{v}}{d t}=\sigma b \tilde{g}(\tilde{u}, \tilde{v}), \quad \tilde{v}(0)=2 \max _{x \in \bar{\Omega}} v_{0}(x) .
$$

Let $\hat{v}=v-\tilde{v}$. Then $\hat{v}(x, 0)<0$ for all $x \in \bar{\Omega}$. We claim that $\hat{v}(x, t)<0$ for all $x \in \bar{\Omega}$ and $t>0$. Indeed, suppose this is not true. Then there exists a $T>0$ such that $\hat{v}(x, t)<0$ for $(x, t) \in \bar{\Omega} \times(0, T)$, and $\hat{v}(x, T)=0$ for some $x \in \bar{\Omega}$. By continuity one has

$$
\max _{x \in \bar{\Omega}} \hat{v}(x, T)=0 .
$$

Now, if there is an $x_{1} \in \Omega$ such that $\hat{v}\left(x_{1}, T\right)=0$, then $\hat{v}_{t}\left(x_{1}, T\right) \geq 0$ and $\Delta \hat{v}\left(x_{1}, T\right) \leq 0$, which yield

$$
-\hat{v}_{t}\left(x_{1}, T\right)+\sigma c \Delta \hat{v}\left(x_{1}, T\right) \leq 0 .
$$

On the other hand, by (1.2) and (2.3),

$$
-\hat{v}_{t}+\sigma c \Delta \hat{v}=\sigma b\left[g(\tilde{u}, \tilde{v})-\left(u-\frac{u v}{1+u^{2}}\right)\right] .
$$


At the point $\left(x_{1}, T\right)$, since $\tilde{v}=v$ and $\tilde{u}>u$, we find that

$$
\begin{aligned}
\tilde{g}(\tilde{u}, \tilde{v}) & =\sup _{C_{1}<\xi<\tilde{u}}\left(\xi-\frac{\xi\left(\tilde{v}-\epsilon_{0}\right)}{1+\xi^{2}}\right)=\sup _{C_{1}<\xi<\tilde{u}}\left(\xi-\frac{\xi\left(v-\epsilon_{0}\right)}{1+\xi^{2}}\right) \\
& >\sup _{C_{1}<\xi<\tilde{u}}\left(\xi-\frac{\xi v}{1+\xi^{2}}\right) \geq \sup _{C_{1}<\xi \leq u}\left(\xi-\frac{\xi v}{1+\xi^{2}}\right) \\
& \geq u-\frac{u v}{1+u^{2}},
\end{aligned}
$$

which gives $-\hat{v}_{t}\left(x_{1}, T\right)+\sigma c \Delta \hat{v}\left(x_{1}, T\right)>0$, leading to a contradiction.

If $\hat{v}(x, T)<0$ for all $x \in \Omega$, then there would be some $x_{1} \in \partial \Omega$ such that $\hat{v}\left(x_{1}, T\right)=0$. Thus the right-hand side of (2.4) is positive at $\left(x_{1}, T\right)$, and by continuity it remains positive in $\Omega^{\prime} \times\{T\}$, where $\Omega^{\prime}$ is a sub-domain of $\Omega$ and $x_{1} \in \partial \Omega^{\prime}$. Therefore, on $\Omega^{\prime} \times\{T\}$ we have

$$
-\hat{v}_{t}+\sigma c \Delta \hat{v} \geq 0 \text {. }
$$

(Note that it is not known whether or not this inequality holds in $\Omega \times(0, T]$, which makes the situation delicate.) Now treating (2.4) as an elliptic equation in $\bar{\Omega}^{\prime} \times\{T\}$, we see by Hopf's boundary lemma that $\partial \hat{v} / \partial \nu\left(x_{1}, T\right)>0$, which contradicts the Neumann boundary condition (1.4). Hence we must have $v(x, t)<\tilde{v}(t)$ for all $x \in \bar{\Omega}$ and $t>0$.

We finally consider the flow defined by the system

$$
\frac{d \tilde{u}}{d t}=\tilde{a}-\tilde{u}, \quad \frac{d \tilde{v}}{d t}=\sigma b \tilde{g}(\tilde{u}, \tilde{v})
$$

in $\Re$. From the definition of $\tilde{g}$ it follows that

$$
\tilde{g}<0 \text { when } \tilde{v}>1+\tilde{u}^{2}+\epsilon_{0} \text {, and } \quad \tilde{g}>0 \text { when } \quad \tilde{v}<1+\tilde{u}^{2}+\epsilon_{0} .
$$

Thus the zero curve of $\tilde{g}$ is given by $\tilde{v}=1+\tilde{u}^{2}+\epsilon_{0}$, and therefore this dynamic system has a unique equilibrium $(\tilde{u}, \tilde{v})=\left(\tilde{a}, 1+\tilde{a}^{2}+\epsilon_{0}\right)$. By the equation of $\tilde{u}$ it is clear that $\lim _{t \rightarrow \infty} \tilde{u}(t)=\tilde{a}$. It is therefore not hard to see that the equilibrium is globally asymptotically stable in $\Re$ and so $\lim _{t \rightarrow \infty} \tilde{v}(t)=1+\tilde{a}^{2}+\epsilon_{0}$. The conclusion of this proposition readily follows since $\tilde{a}<a, \tilde{a}^{2}+\epsilon_{0}<a^{2}$, and $u$ and $v$ are respectively bounded above by $\tilde{u}$ and $\tilde{v}$.

\section{Properties of nonconstant steady states}

In this section, we shall discuss the basic properties of non-homogeneous steady states of the Lengyel-Epstein reaction-diffusion system; namely, positive nonconstant solutions to the elliptic system

$$
\begin{aligned}
\Delta u+a-u-\frac{4 u v}{1+u^{2}} & =0, \\
d \Delta v+u-\frac{u v}{1+u^{2}} & =0
\end{aligned}
$$

$(d=c / b)$, subject to the homogeneous Neumann boundary condition

$$
\partial u / \partial \nu=\partial v / \partial \nu=0, x \in \partial \Omega .
$$

Proposition 3.1 (A priori estimates). If $(u, v)=(u(x), v(x))$ is a positive solution to the boundary value problem (3.1)-(3.3), then

$$
\frac{a}{5+4 a^{2}}<u<a \quad \text { and } 1+\left(\frac{a}{5+4 a^{2}}\right)^{2}<v<1+a^{2}, \quad x \in \Omega .
$$


The upper bounds here of course follow by Proposition 2.2 directly, which however does not yield the lower bounds. One could possibly derive the lower bounds in (3.4) by using the comparison argument as in Section 2, but we prefer a simpler and more direct proof based on an elliptic maximum principle due to Lou and $\mathrm{Ni}$ [LN].

Lemma 3.1 ( $(\overline{\mathrm{LN}})$. Suppose that $F(x, w) \in C(\bar{\Omega} \times R)$. If $w \in C^{2}(\Omega) \cap C^{1}(\bar{\Omega})$ satisfies

$$
\Delta w(x)+F(x, w(x)) \geq 0 \quad \text { in } \quad \Omega, \quad \partial w / \partial \nu \leq 0 \quad \text { on } \quad \partial \Omega
$$

and $w\left(x_{0}\right)=\max _{\bar{\Omega}} w$, then $F\left(x_{0}, w\left(x_{0}\right)\right) \geq 0$. Similarly, if the two inequalities in (3.5) are reversed and $w\left(x_{0}\right)=\min _{\bar{\Omega}} w$, then $F\left(x_{0}, w\left(x_{0}\right)\right) \leq 0$.

Proof of Proposition 3.1. If at some point in $\bar{\Omega}$ the function $u$ attains its maximum over $\bar{\Omega}$, then at this point we have

$$
a-u-4 u v /\left(1+u^{2}\right) \geq 0,
$$

by (3.1), (3.3) and Lemma 3.1 It readily follows that $u<a$. Similarly, if $v$ attains a maximum over $\bar{\Omega}$ at some point, then by (3.2), (3.3) and Lemma 3.1 we have

$$
u-u v /\left(1+u^{2}\right) \geq 0,
$$

implying that $v \leq 1+u^{2}<1+a^{2}$.

If $u$ attains its minimum over $\bar{\Omega}$ at a point, then

$$
a \leq u+4 u v /\left(1+u^{2}\right)<u+4 u\left(1+a^{2}\right)=\left(5+4 a^{2}\right) u,
$$

and so $u>a /\left(5+4 a^{2}\right)$. Similarly, we can derive the lower bound for $v$.

For a given pair of solutions $u=u(x), v=v(x)$ to the elliptic problem (3.1)(3.3), we denote their averages over $\Omega$ by

$$
\bar{u}=\frac{1}{|\Omega|} \int_{\Omega} u(x) d x, \quad \bar{v}=\frac{1}{|\Omega|} \int_{\Omega} v(x) d x
$$

where $|\Omega|$ is the volume of $\Omega$.

Lemma 3.2. $\bar{u}=a / 5$.

Proof. Write

$$
w(x)=4 d v(x)-u(x) .
$$

By (3.1) and (3.2) we obtain

$$
\Delta w-a+5 u=0 .
$$

Integrating (3.6) over $\Omega$ gives

$$
\int_{\Omega}(5 u-a) d x=\int_{\Omega} \Delta w d x=\int_{\partial \Omega} \frac{\partial w}{\partial \nu} d S=0
$$

by the boundary condition (3.3). Hence $\bar{u}=a / 5$.

Let

$$
\phi=u-\bar{u}, \quad \psi=v-\bar{v} .
$$

Then $\int \phi=\int \psi=0$. (Here and in the sequel we use the notation $\int$ to denote the integral over $\Omega$ ). If $(u, v)$ is not a constant solution, then $\phi$ and $\psi$ are non-trivial and must change sign in $\Omega$. Our next result shows that, however, the product $\phi \psi$ has a positive average over $\Omega$. 
Lemma 3.3. Suppose $u$ and $v$ are nonconstant solutions of (3.1)-(3.3). Then $\int \phi \psi>0$ and $\int \nabla \phi \cdot \nabla \psi>0$.

Proof. Rewrite (3.6) as

$$
-\Delta w=5 \phi .
$$

Multiply this by $w=4 d v-u$ and integrate by parts yielding

$$
\begin{aligned}
\int|\nabla w|^{2} & =\int 5 \phi w \\
& =20 d \int \phi v-5 \int \phi u \\
& =20 d \int \phi \psi-5 \int \phi^{2}
\end{aligned}
$$

since $\phi=u-\bar{u}, \psi=v-\bar{v}$ and $\int \phi=\int \psi=0$. Thus we obtain

$$
\int \phi \psi=\frac{1}{20 d}\left(\int|\nabla w|^{2}+5 \int \phi^{2}\right) .
$$

If we multiply (3.6) by $\phi$ and integrate by parts, then

$$
5 \int \phi^{2}=\int \nabla \phi \cdot \nabla w=4 d \int \nabla \phi \cdot \nabla \psi-\int|\nabla \phi|^{2},
$$

which implies that

$$
\int \nabla \phi \cdot \nabla \psi=\frac{1}{4 d}\left(\int|\nabla \phi|^{2}+5 \int \phi^{2}\right) .
$$

The conclusion of this lemma follows from (3.7) and (3.8) immediately.

The estimate (3.4) guarantees that there exists a constant $c_{g}$ depending on $a$ only such that

This enables us to derive

$$
|g(u, v)|=\left|u-u v /\left(1+u^{2}\right)\right| \leq c_{g} .
$$

Lemma 3.4. There exists a constant $C_{g}$, depending on a and $\Omega$, such that

$$
\int \psi^{2}+\int|\nabla \psi|^{2} \leq C_{g} d^{-2}
$$

Proof. By (3.2) and the Schwarz inequality we have

$$
d \int|\nabla \psi|^{2}=\int g(u, v) \psi \leq c_{g} \int|\psi| \leq c_{g} \sqrt{|\Omega|}\left(\int|\psi|^{2}\right)^{1 / 2} .
$$

Applying the well-known Poincaré inequality

$$
\int \psi^{2} \leq \frac{1}{\lambda_{1}} \int|\nabla \psi|^{2}
$$

where $\lambda_{1}>0$ is the first positive eigenvalue of $-\Delta$ on $\Omega$ subject to the Neumann boundary condition, we obtain

$$
d \int|\nabla \psi|^{2} \leq c_{g} \sqrt{|\Omega| / \lambda_{1}}\left(\int|\nabla \psi|^{2}\right)^{1 / 2} .
$$

This gives

$$
\int|\nabla \psi|^{2} \leq \frac{c_{g}^{2}|\Omega|}{\lambda_{1}} d^{-2}
$$


Applying the Poincaré inequality again we obtain (3.9) with

$$
C_{g}=c_{g}^{2}|\Omega|\left(1+\lambda_{1}\right) / \lambda_{1}^{2} .
$$

Lemma 3.5. Let $(u, v)$ be a nonconstant solution of the problem 3.1)-3.3. Then

$$
\frac{12 \lambda_{1}^{2}}{3 \lambda_{1}^{2}+15 \lambda_{1}+25}<\frac{\int|\nabla u|^{2}}{d^{2} \int|\nabla v|^{2}}<16 .
$$

Proof. Recall that $w=4 d v-u$. By (3.8) we have

$$
\begin{aligned}
\int|\nabla w|^{2} & =16 d^{2} \int|\nabla \psi|^{2}-8 d \int \nabla \phi \cdot \nabla \psi+\int|\nabla \phi|^{2} \\
& =16 d^{2} \int|\nabla \psi|^{2}-\int|\nabla \phi|^{2}-10 \int \phi^{2}
\end{aligned}
$$

and so

$$
\int|\nabla \phi|^{2}+10 \int \phi^{2} \leq 16 d^{2} \int|\nabla \psi|^{2} .
$$

The second inequality of (3.10) thus follows.

To finish the proof, we first claim

$$
4 d^{2} \int|\nabla \psi|^{2}=\frac{1}{4} \int|\nabla \phi|^{2}+\frac{5}{4} \int \phi^{2}+5 d \int \phi \psi .
$$

Indeed, continuing the calculation above we find that

$$
16 d^{2} \int|\nabla \psi|^{2}=\int|\nabla w|^{2}+\int|\nabla \phi|^{2}+10 \int \phi^{2}
$$

and using (3.7) we get

$$
16 d^{2} \int|\nabla \psi|^{2}=\int|\nabla \phi|^{2}+5 \int \phi^{2}+20 d \int \phi \psi,
$$

which is exactly (3.12).

We next recall the elementary Cauchy inequality: for any given real numbers $\xi$, $\zeta$ and $\kappa>0$,

$$
\xi \zeta \leq \frac{1}{4 \kappa} \xi^{2}+\kappa \zeta^{2} .
$$

Now, from (3.12) and (3.13) it follows that

$$
\begin{aligned}
4 d^{2} \int|\nabla \psi|^{2} & \leq \frac{1}{4} \int|\nabla \phi|^{2}+\frac{5}{4} \int \phi^{2}+\frac{25}{12 \lambda_{1}} \int \phi^{2}+3 d^{2} \lambda_{1} \int \psi^{2} \\
& \leq\left[\frac{1}{4}+\left(\frac{5}{4}+\frac{25}{12 \lambda_{1}}\right) / \lambda_{1}\right] \int|\nabla \phi|^{2}+3 d^{2} \int|\nabla \psi|^{2} .
\end{aligned}
$$

Hence

$$
d^{2} \int|\nabla \psi|^{2} \leq\left[\frac{1}{4}+\left(\frac{5}{4}+\frac{25}{12 \lambda_{1}}\right) / \lambda_{1}\right] \int|\nabla \phi|^{2},
$$

establishing the first inequality of (3.10).

Lemma 3.6. Let $(u, v)$ be a nonconstant solution of the problem (3.1)-(3.3). Then

$$
\frac{12 \lambda_{1}^{3}}{\left(\lambda_{1}+1\right)\left(3 \lambda_{1}^{2}+15 \lambda_{1}+25\right)}<\frac{\int\left(|\nabla \phi|^{2}+\phi^{2}\right)}{d^{2} \int\left(|\nabla \psi|^{2}+\psi^{2}\right)}<16 .
$$


Proof. By the Poincaré inequality we have

$$
\int\left(|\nabla \psi|^{2}+\psi^{2}\right) \leq\left(\lambda_{1}+1\right) / \lambda_{1} \int|\nabla \psi|^{2} .
$$

This, together with the first inequality of (3.10), implies the first inequality of (3.15). The second inequality of (3.15) follows from (3.11).

\section{Non-existence of TURing PATterns}

Several non-existence results for the nonconstant steady state solutions will be given in this section. In brief, our theorems show that the chemical reaction creates a Turing pattern only if the parameter $a$ (related to the feed concentrations), the size of the reactor (reflected by its first eigenvalue $\lambda_{1}$ ), and the effective diffusion rate $d=c / b$ are suitably large.

The non-existence results also play a critical role in obtaining the existence theorem for larger parameters in Section 6.

Theorem 1. There is a constant $d_{0}=d_{0}\left(a, \lambda_{1}\right)>0$ such that the problem (3.1)(3.3) does not admit a nonconstant solution for $0<d<d_{0}$.

Proof. Multiplying (3.2) by $\psi$ and integrating by parts we derive

$$
\begin{aligned}
d \int|\nabla \psi|^{2} & =\int \phi \psi-\int \frac{u v \psi}{1+u^{2}} \\
& =\int \phi \psi-\int\left(\frac{u v}{1+u^{2}}-\frac{u \bar{v}}{1+u^{2}}\right) \psi-\int\left(\frac{u \bar{v}}{1+u^{2}}-\frac{\bar{u} \bar{v}}{1+\bar{u}^{2}}\right) \psi \\
& =\int \phi \psi-\int \frac{u}{1+u^{2}} \psi^{2}+\int \frac{(u \bar{u}-1) \bar{v}}{\left(1+u^{2}\right)\left(1+\bar{u}^{2}\right)} \phi \psi .
\end{aligned}
$$

From the a priori estimates in Proposition 3.1 it follows that

$$
\begin{aligned}
d \int|\nabla \psi|^{2} & \leq C_{1}(a) \int|\phi \psi|-\int \frac{u}{1+u^{2}} \psi^{2} \\
& \leq C_{1}(a) \int|\phi \psi|-C_{2}(a) \int \psi^{2} .
\end{aligned}
$$

Applying the Cauchy inequality and the Poincaré inequality we have

$$
d \int|\nabla \psi|^{2} \leq \frac{C_{1}^{2}}{4 C_{2}} \int \phi^{2} \leq \frac{C_{1}^{2}}{4 C_{2} \lambda_{1}} \int|\nabla \phi|^{2},
$$

which, in turn, implies that

$$
\int|\nabla \psi|^{2} \leq \frac{d}{d_{0}} \int|\nabla \psi|^{2}
$$

by (3.10), where

$$
d_{0}=d_{0}\left(a, \lambda_{1}\right)=\frac{C_{2}(a) \lambda_{1}}{4 C_{1}^{2}(a)} .
$$

Clearly, if $d<d_{0}$, then $\int|\nabla \psi|^{2}=0$ and thus $\int|\nabla \phi|^{2}=0$ by (3.10) again. Therefore $|\nabla \phi| \equiv|\nabla \psi| \equiv 0$ over $\Omega$, and $u$ and $v$ must be constants.

We remark that for a given $a$ it is very involved to get a good estimate for the constant $d_{0}$ derived above. Nevertheless, if $a$ is not very large, then we can obtain a much simpler estimate using a different approach. 
Theorem 2. If $a^{2} \leq 75$, then the boundary value problem (3.1)-3.3) does not admit any nonconstant solution if

$$
\frac{1}{d}>\frac{8 a}{5}-\frac{25}{a} .
$$

In particular, there is no nonconstant solution for all $d>0$ if $a^{2} \leq 125 / 8$.

Proof. If we multiply (3.1) by $\left(1+u^{2}\right) \phi$, then

$$
\phi \Delta \phi+u^{2} \phi \Delta \phi+(a-\bar{u}-\phi) \phi+(a-u) u^{2} \phi-4 u v \phi=0 .
$$

Integration by parts gives

$$
\begin{aligned}
\int|\nabla \phi|^{2}= & -\int \nabla\left(u^{2} \phi\right) \cdot \nabla \phi-\int \phi^{2}+\int(a-u) u^{2} \phi-4 \int u v \phi \\
= & -\int\left(2 u \phi+u^{2}\right)|\nabla \phi|^{2}-\int \phi^{2}+\int \phi\left[(a-u) u^{2}-\frac{4}{125} a^{3}\right] \\
& \quad-4 \int \phi(u v-\bar{u} \bar{v}) \\
= & \frac{1}{5} \int(2 a-15 u) u|\nabla \phi|^{2}+\int \phi^{2}\left(-u^{2}+\frac{4 a}{5} u+\frac{4}{25} a^{2}-1\right) \\
& -4 \int v \phi^{2}-\frac{4 a}{5} \int \phi \psi .
\end{aligned}
$$

As

$$
(2 a-15 u) u \leq \frac{a^{2}}{15}, \quad-u^{2}+\frac{4 a}{5} u+\frac{4}{25} a^{2} \leq \frac{8 a^{2}}{25}
$$

and $v \geq 1$, this leads to

$$
\int|\nabla \phi|^{2} \leq \frac{a^{2}}{75} \int|\nabla \phi|^{2}+\left(\frac{8 a^{2}}{25}-5\right) \int \phi^{2}-\frac{4 a}{5} \int \phi \psi
$$

Applying (3.7) to $\int \phi \psi$ we obtain

$$
\int|\nabla \phi|^{2} \leq \frac{a^{2}}{75} \int|\nabla \phi|^{2}+\left(\frac{8 a^{2}}{25}-5-\frac{a}{5 d}\right) \int \phi^{2} .
$$

Now, if $a^{2} \leq 75$ and (4.3) holds, then for a nonconstant solution $(u, v)$, we conclude that $\int|\nabla \phi|^{2}<\int|\nabla \phi|^{2}$, a contradiction. Thus $\phi \equiv 0$.

If we fix $a$ and $d$, then Theorem 1 also gives the non-existence for large $\lambda_{1}$. To see this, recall that $d_{0}=C(a) \lambda_{1}$ for some constant $C(a)$, so taking $\lambda_{1}$ suitably large one can make $d<d_{0}$.

Moreover, our next theorem provides a stronger result than the observation above: If $\lambda_{1}$ is sufficiently large, then the non-existence holds for all $d>0$.

Theorem 3. There is a constant $\Lambda=\Lambda(a)>0$ such that the problem (3.1)-3.3) does not admit any nonconstant solution if $\lambda_{1}(\Omega)>\Lambda$.

Proof. Multiplying equation (3.1) by $\phi$ and integrating by parts we have

$$
\begin{aligned}
\int|\nabla \phi|^{2} & =\int\left(a-u-\frac{4 u v}{1+u^{2}}\right) \phi \\
& =-\int \phi^{2}-4 \int \frac{u v}{1+u^{2}} \phi
\end{aligned}
$$


Since

$$
\begin{aligned}
& \int \frac{u v}{1+u^{2}} \phi \\
= & \int\left(\frac{u v}{1+u^{2}}-\frac{\bar{u} v}{1+u^{2}}+\frac{\bar{u} v}{1+u^{2}}-\frac{\bar{u} \bar{v}}{1+u^{2}}+\frac{\bar{u} \bar{v}}{1+u^{2}}-\frac{\bar{u} \bar{v}}{1+\bar{u}^{2}}\right) \phi \\
= & \int \frac{v}{1+u^{2}} \phi^{2}+\int \frac{\bar{u}}{1+u^{2}} \phi \psi-\int \frac{\bar{u} \bar{v}(\bar{u}+u)}{\left(1+u^{2}\right)\left(1+\bar{u}^{2}\right)} \phi^{2},
\end{aligned}
$$

it follows that

$$
\int|\nabla \phi|^{2} \leq \int \frac{4 \bar{u} \bar{v}(\bar{u}+u)}{\left(1+u^{2}\right)\left(1+\bar{u}^{2}\right)} \phi^{2}-\int \frac{4 \bar{u}}{1+u^{2}} \phi \psi .
$$

Applying the a priori estimates in Proposition 3.1 we obtain the estimate

$$
\int|\nabla \phi|^{2} \leq C \int \phi^{2}+C \int|\phi \psi|
$$

where $C$ stands for a generic constant depending on $a$ in this proof, which may differ from line to line. By the Schwarz inequality and the Poincaré inequality we have

$$
\int|\phi \psi| \leq\left(\int|\phi|^{2}\right)^{1 / 2}\left(\int|\psi|^{2}\right)^{1 / 2} \leq \frac{1}{\lambda_{1}}\left(\int|\nabla \phi|^{2}\right)^{1 / 2}\left(\int|\nabla \psi|^{2}\right)^{1 / 2} .
$$

Consequently,

$$
\int|\phi \psi| \leq C \lambda_{1}^{-3 / 2} d^{-1 / 2} \int|\nabla \phi|^{2}
$$

by (4.1). Combining this with (4.5) we find that

$$
\int|\nabla \phi|^{2} \leq \frac{C}{\lambda_{1}}\left(1+\frac{1}{\left(\lambda_{1} d\right)^{1 / 2}}\right) \int|\nabla \phi|^{2} .
$$

Now, if $d$ is not small, say, $d \geq 1$, then by choosing $\Lambda$ so large that for $\lambda_{1}>\Lambda$,

$$
\frac{C}{\lambda_{1}}\left(1+\lambda_{1}^{-1 / 2}\right)<1
$$

we can make by (4.6) $\int|\nabla \phi|^{2}=0$, which forces $u$ and $v$ to be constants. On the other hand, if $d<1$, then we can choose $\lambda_{1}$ so large that $d_{0}>1$, which gives the non-existence again by Theorem 1

\section{TURING INSTABILITY}

Let

$$
f(u, v)=a-u-\frac{4 u v}{1+u^{2}}, \quad g(u, v)=u-\frac{u v}{1+u^{2}} .
$$

Then the Lengyel-Epstein system (1.1)-(1.2) can be written as

$$
u_{t}=\Delta u+f(u, v), \quad v_{t}=\sigma[c \Delta v+b g(u, v)] .
$$

A constant steady-state solution $\left(u^{*}, v^{*}\right)$ of this system subject to the Neumann boundary condition (1.4) satisfies $f\left(u^{*}, v^{*}\right)=g\left(u^{*}, v^{*}\right)=0$. Hence $\left(u^{*}, v^{*}\right)$ is unique and is given by

$$
\left(u^{*}, v^{*}\right)=\left(\alpha, 1+\alpha^{2}\right), \quad \alpha=a / 5 .
$$


Observe that $f_{v}\left(u^{*}, v^{*}\right)<0, g_{u}\left(u^{*}, v^{*}\right)>0$ and $g_{v}\left(u^{*}, v^{*}\right)<0$. If

$$
f_{u}\left(u^{*}, v^{*}\right)=\left(3 \alpha^{2}-5\right) /\left(1+\alpha^{2}\right)>0,
$$

then we call $u$ an activator, $v$ an inhibitor, and the system (1.1)-(1.2) an activatorinhibitor system. Clearly this is fulfilled if

$$
3 \alpha^{2}-5>0 .
$$

We say that this constant solution is Turing unstable if it is stable in the absence of diffusion, and it becomes unstable when diffusion is present. More precisely, this requires the following two conditions:

(i) It is stable as an equilibrium of the system of ordinary differential equations

$$
\frac{d u}{d t}=f(u, v), \quad \frac{d v}{d t}=\sigma b g(u, v) .
$$

(ii) It is unstable as a steady state of the reaction-diffusion equations (1.1)-(1.2) subject to the homogeneous Neumann boundary conditions.

Using the ODE theory we can easily find a sufficient and necessary condition for (i). The Jacobian matrix of (5.3) evaluated at $\left(u^{*}, v^{*}\right)$ is

$$
J=\left[\begin{array}{cc}
f_{0} & f_{1} \\
\sigma b g_{0} & \sigma b g_{1}
\end{array}\right]
$$

where

$$
f_{0}=\frac{3 \alpha^{2}-5}{1+\alpha^{2}}, \quad f_{1}=-\frac{4 \alpha}{1+\alpha^{2}}, \quad g_{0}=\frac{2 \alpha^{2}}{1+\alpha^{2}}, \quad g_{1}=-\frac{\alpha}{1+\alpha^{2}} .
$$

Since

$$
\frac{\operatorname{det} J}{\sigma b}=f_{0} g_{1}-f_{1} g_{0}=5 \alpha /\left(1+\alpha^{2}\right)>0,
$$

the eigenvalues of $J$ have negative real parts, and so $\left(u^{*}, v^{*}\right)$ is diffusion free stable, if and only if trace $J=f_{0}+\sigma b g_{1}<0$, or equivalently

$$
3 \alpha^{2}-5<\sigma \alpha b \text {. }
$$

Combining (5.2) and (5.6), we find that the condition

(H) $\quad 0<3 \alpha^{2}-5<\sigma \alpha b$

makes the model (1.1)-(1.2) a diffusion-free stable activator-inhibitor system.

We next derive a sufficient condition for (ii) above. Let $0=\lambda_{0}<\lambda_{1} \leq \lambda_{2} \leq$ $\lambda_{3} \leq \cdots$ be the sequence of eigenvalues for the elliptic operator $-\Delta$ subject to the Neumann boundary condition on $\Omega$, where each $\lambda_{i}$ has multiplicity $m_{i} \geq 1$. Let $\phi_{i j}, 1 \leq j \leq m_{i}$, be the normalized eigenfunctions corresponding to $\lambda_{i}$. Then the set $\left\{\phi_{i j}\right\}, i \geq 0,1 \leq j \leq m_{i}$, forms a complete orthonormal basis in $L^{2}(\Omega)$. If

$$
\lambda_{1}<f_{0}=\frac{3 \alpha^{2}-5}{1+\alpha^{2}}
$$

then we define $i_{\alpha}=i_{\alpha}(\alpha, \Omega)$ to be the largest positive integer such that

$$
\lambda_{i}<f_{0} \quad \text { for } \quad i \leq i_{\alpha} .
$$

Clearly, if (5.7) is satisfied, then $1 \leq i_{\alpha}<\infty$. In this case, we let

$$
\tilde{d}=\tilde{d}(\alpha, \Omega)=\min _{1 \leq i \leq i_{\alpha}} d_{i}, \quad d_{i}=\frac{\alpha}{1+\alpha^{2}} \frac{\lambda_{i}+5}{\lambda_{i}\left(f_{0}-\lambda_{i}\right)} .
$$


Then the local stability of $\left(u^{*}, v^{*}\right)$ can be summarized as follows:

Lemma 5.1. Assume $(\mathrm{H})$ holds. If $\lambda_{1} \geq f_{0}$, or $\lambda_{1}<f_{0}$ and $0<d=c / b<\tilde{d}$, then the constant steady state $\left(u^{*}, v^{*}\right)$ is asymptotically stable. If $\lambda_{1}\left\langle f_{0}\right.$, and $\left.d\right\rangle \tilde{d}$, then $\left(u^{*}, v^{*}\right)$ is unstable.

Proof. Consider the linearization operator

$$
L_{\sigma}=\left(\begin{array}{cc}
\Delta+f_{0} & f_{1} \\
\sigma b g_{0} & \sigma c \Delta+\sigma b g_{1}
\end{array}\right)
$$

where $f_{0}, f_{1}, g_{0}$ and $g_{1}$ are given in (5.4). From the standard linear operator theory (or Theorem 1 of $[\mathrm{CH}]$ ) it is known that if all the eigenvalues of this operator have negative real parts, then $\left(u^{*}, v^{*}\right)$ is asymptotically stable, and if some eigenvalues have positive real parts, the $\left(u^{*}, v^{*}\right)$ is unstable.

Suppose $(\phi(x), \psi(x))$ is an eigenfunction of $L_{\sigma}$ corresponding to an eigenvalue $\mu$. Then

$$
\left(\Delta \phi+\left(f_{0}-\mu\right) \phi+f_{1} \psi, \sigma c \Delta \psi+\sigma b g_{0} \phi+\left(\sigma b g_{1}-\mu\right) \psi\right)=(0,0) .
$$

Letting

$$
\phi=\sum_{0 \leq i \leq \infty, 1 \leq j \leq m_{i}} a_{i j} \phi_{i j} \text { and } \quad \psi=\sum_{0 \leq i \leq \infty, 1 \leq j \leq m_{i}} b_{i j} \phi_{i j},
$$

we find that

$$
\sum_{0 \leq i \leq \infty, 1 \leq j \leq m_{i}}\left(\begin{array}{cc}
f_{0}-\lambda_{i}-\mu & f_{1} \\
\sigma b g_{0} & \sigma b g_{1}-\sigma c \lambda_{i}-\mu
\end{array}\right)\left(\begin{array}{l}
a_{i j} \\
b_{i j}
\end{array}\right) \phi_{i j}=0 .
$$

It readily follows that $\mu$ is an eigenvalue of $L_{\sigma}$ if and only if for some $i \geq 0$ the determinant of the matrix is zero, that is,

$$
\mu^{2}+P_{i} \mu+\sigma b Q_{i}=0
$$

where

$$
P_{i}=(1+\sigma c) \lambda_{i}-\left(f_{0}+\sigma b g_{1}\right)=(1+\sigma c) \lambda_{i}-\left(3 \alpha^{2}-5-\sigma \alpha b\right) /\left(1+\alpha^{2}\right)>0
$$

by (5.4) and condition $(\mathrm{H})$, and

$$
Q_{i}=\left(f_{0}-\lambda_{i}\right)\left(g_{1}-d \lambda_{i}\right)-f_{1} g_{0}=d \lambda_{i}\left(\lambda_{i}-f_{0}\right)+\frac{\alpha}{1+\alpha^{2}}\left(\lambda_{i}+5\right)
$$

since $c=b d$ and $f_{0} g_{1}-f_{1} g_{0}=5 \alpha /\left(1+\alpha^{2}\right)$. Clearly, $Q_{0}>0$ for $\lambda_{0}=0$. Now, if $\lambda_{1} \geq f_{0}$, then by (5.9) $Q_{i}>0$ for all $i \geq 1$. This implies that Re $\mu<0$ for all eigenvalues $\mu$, and so the steady-state $\left(u^{*}, v^{*}\right)$ is asymptotically stable.

If $\lambda_{1}<f_{0}$ and $0<d<\tilde{d}$, then

$$
\lambda_{i}<f_{0} \quad \text { and } \quad d<d_{i}, \quad i \in\left[1, i_{\alpha}\right] .
$$

It follows that $Q_{i}>0$ for all $i \in\left[1, i_{\alpha}\right]$. Furthermore, if $i>i_{\alpha}$, then $\lambda_{i} \geq f_{0}$ and $Q_{i}>0$ by (5.9). The argument leads to the asymptotical stability of $\left(u^{*}, v^{*}\right)$ again.

Finally, if $\lambda_{1}<f_{0}$, and $d>\tilde{d}$, then we may assume that the minimum in (5.8) is attained by $k \in\left[1, i_{\alpha}\right]$. Thus

$$
d>d_{k}
$$

which implies $Q_{k}<0$, and so the instability of $\left(u^{*}, v^{*}\right)$ follows. 


\section{Existence of NON-HOMOGEneOUs SteAdy States}

In view of the non-existence results for small diffusion coefficients in Section 4, it is particularly interesting to know if (1.1)-(1.4) admit non-homogeneous steady states when the system parameters take on larger values. We shall give a positive answer to this question in this section, using a standard approach based on the Leray-Schauder degree theory for compact operators in Banach space.

We first reformulate the system 3.1 3.3 in the framework that the degree theory can be easily applied. Let $\tilde{u}=u-\alpha, \tilde{v}=v-1-\alpha^{2}$. Then (3.1)-3.2 is shifted to

$$
\begin{aligned}
-\Delta \tilde{u} & =f_{0} \tilde{u}+f_{1} \tilde{v}+f_{2}(\tilde{u}, \tilde{v}), \\
-d \Delta \tilde{v} & =g_{0} \tilde{u}+g_{1} \tilde{v}+g_{2}(\tilde{u}, \tilde{v}),
\end{aligned}
$$

where $f_{2}$ and $g_{2}$ consist of higher order terms of $\tilde{u}$ and $\tilde{v}$. The constant steady state $\left(\alpha, 1+\alpha^{2}\right)$ is thus shifted to $(0,0)$, and the region $\Re_{a}$ defined in Section 2 becomes the rectangle

$$
S=\left\{(\tilde{u}, \tilde{v}):-\alpha<\tilde{u}<4 \alpha,-1-\alpha^{2}<\tilde{v}<24 \alpha^{2}\right\}
$$

For $w \in C^{\beta}(\bar{\Omega}), \beta \in(0,1)$, let $u=G(w)$ be the solution to the boundary value problem of the linear elliptic equation

$$
-\Delta u+f_{0} u=w \quad \text { in } \Omega, \quad \frac{\partial u}{\partial \nu}=0 \quad \text { on } \quad \partial \Omega,
$$

and let $v=G_{d}(w)$ be the solution to the linear problem

$$
-d \Delta v-g_{1} v=w \quad \text { in } \Omega, \quad \frac{\partial v}{\partial \nu}=0 \quad \text { on } \quad \partial \Omega,
$$

where $f_{0}=\left(3 \alpha^{2}-5\right) /\left(1+\alpha^{2}\right)>0$ and $g_{1}=-\alpha /\left(1+\alpha^{2}\right)<0$. By the standard existence theory of linear elliptic equations, $u$ and $v$ are uniquely defined, and by the Schauder estimates $u, v \in C^{2+\beta}(\Omega)$ and the mappings $G$ and $G_{d}$ are continuous and compact. Let

$$
E=\left\{(u, v): u, v \in C^{1+\beta}(\bar{\Omega}), \quad \frac{\partial u}{\partial \nu}=\frac{\partial v}{\partial \nu}=0 \quad \text { on } \quad \partial \Omega\right\},
$$

and $U=(\tilde{u}, \tilde{v})$. Then the problem $3.1-3.3)$ can be interpreted as the equation

$$
U=K(d) U+H(U)
$$

in $E$, where

$$
K(d) U=\left(2 f_{0} G(\tilde{u})+f_{1} G(\tilde{v}), g_{0} G_{d}(\tilde{u})\right)
$$

is a compact linear operator on $E$ for any given $d>0$, and

$$
H(U)=\left(G\left(f_{2}\right), G_{d}\left(g_{2}\right)\right)=o(|U|)
$$

for $U$ near zero uniformly on closed $d$ sub-intervals of $(0, \infty)$, and is a compact operator on $E$ as well.

Theorem 4. Assume $(\mathrm{H})$ holds. Suppose $\lambda_{1}<f_{0}<\lambda_{2}$, and $\lambda_{1}$ has an odd multiplicity. Then for any $d>d_{1}$ the problem (3.1)-(3.3) possesses at least one nonconstant positive solution. 
Proof. By Proposition 2.2 or Proposition 3.1, equation (6.1) has no solution on the boundary of $S$. Thus the Leray-Schauder degree $\operatorname{deg}(I-K(d)-H, E \cap S, 0)$ is well defined, and by the homotopy invariance it is a constant for all $d>0$. We claim that

$$
\operatorname{deg}(I-K(d)-H, E \cap S, 0)=1, \quad d>0 .
$$

To prove (6.2), we recall that if $(0,0)$ is an isolated zero of $I-K(d)-H$ and the linear operator $I-K(d)$ is a bijection, then

$$
\operatorname{deg}(I-K(d)-H, E \cap S, 0)=i(I-K(d),(0,0))=(-1)^{p},
$$

where $p$ is the sum of the algebraic multiplicities of the positive eigenvalues of $K(d)-$ $I$. Note that if $\mu$ is an eigenvalue of $K(d)-I$ with a corresponding eigenfunction $(\phi, \psi)$, then

$$
\begin{aligned}
-(\mu+1) \Delta \phi & =(-\mu+1) f_{0} \phi+f_{1} \psi, \\
-d(\mu+1) \Delta \psi & =g_{0} \phi+(\mu+1) g_{1} \psi .
\end{aligned}
$$

As in the proof of Lemma 5.1 we use the Fourier expansion

$$
\phi=\sum_{0 \leq i \leq \infty, 1 \leq j \leq m_{i}} a_{i j} \phi_{i j} \text { and } \quad \psi=\sum_{0 \leq i \leq \infty, 1 \leq j \leq m_{i}} b_{i j} \phi_{i j} .
$$

Then $\sum_{0 \leq i \leq \infty, 1 \leq j \leq m_{i}} B_{i}\left(\begin{array}{c}a_{i j} \\ b_{i j}\end{array}\right) \phi_{i j}=0$, where

$$
B_{i}=\left(\begin{array}{cc}
(-\mu+1) f_{0}-(\mu+1) \lambda_{i} & f_{1} \\
g_{0} & (\mu+1) g_{1}-d(\mu+1) \lambda_{i}
\end{array}\right) .
$$

Hence the set of eigenvalues of $K(d)-I$ consists exactly of all roots of the characteristic equation

$$
\left(f_{0}+\lambda_{i}\right) \mu^{2}+2 \lambda_{i} \mu+\lambda_{i}-f_{0}-\frac{f_{1} g_{0}}{d \lambda_{i}-g_{1}}=0
$$

where the integer $i$ runs from zero to $\infty$. By (5.9) we find that here the constant term is

$$
\lambda_{i}-f_{0}-\frac{f_{1} g_{0}}{d \lambda_{i}-g_{1}}=\frac{Q_{i}}{d \lambda_{i}-g_{1}} .
$$

Since $f_{0}>0$ and $g_{1}<0$, it is clear that $K\left(d_{0}\right)-I$ and the operator $L_{\sigma}$ defined in Section 5 have the same number of positive eigenvalues.

By Theorem 1 we may find $d_{0}>0$ sufficiently small such that (6.1) has no solution other than $(0,0)$ in $S$. By Lemma 5.1 and the discussion above we can make the linear operator $I-K\left(d_{0}\right)$ a bijection with no positive eigenvalues. Hence from (6.3) it follows that

$$
\operatorname{deg}(I-K(d)-H, E \cap S, 0)=i\left(I-K\left(d_{0}\right),(0,0)\right)=1,
$$

and (6.2) is established.

Now suppose for contradiction that there exists $d>d_{1}$ such that (3.1)-(3.3) does not admit any nonconstant solution under our assumption. Then $(0,0)$ is an isolated zero of $I-K(d)-H$ in $S$. We shall show that this would imply that the index $i(I-K(d),(0,0))=-1$, which gives the desired contradiction to (6.2) and completes our proof.

Since $f_{0}>\lambda_{1}$, we find that $Q_{1}<0$ for all $d>d_{1}$. Let $i=1$, then (6.4) has one positive root, say, $\mu_{1}>0$, and a negative root. With our further assumption that 
$f_{0}<\lambda_{2}$, we have $Q_{i}>0$ for all $i \geq 2$. Therefore, when $i \geq 2$, the characteristic equation (6.4) has no roots with non-negative real parts. We conclude that $K(d)-I$ has exactly one positive eigenvalue $\mu_{1}>0$, and all other eigenvalues have negative real parts. Thus (6.3) is valid for all $d>d_{1}$, with $p$ being the algebraic multiplicity of $\mu_{1}$ :

$$
p=\operatorname{dim} \bigcup_{i=1}^{\infty} \operatorname{ker} A^{i}, \quad A=K(d)-\left(\mu_{1}+1\right) I .
$$

Using some standard argument we shall prove $p=m_{1}$, which clearly yields $i(I-K(d),(0,0))=-1$ since $m_{1}$ is assumed to be an odd number. Indeed, as $\mu_{1}$ is an eigenvalue of $K(d)-I$, the discussion above shows that

$$
\operatorname{ker} A=\left\{\left(\begin{array}{c}
f_{1} \\
\left(\mu_{1}-1\right) f_{0}+\left(\mu_{1}+1\right) \lambda_{1}
\end{array}\right) \phi_{1 j}, 1 \leq j \leq m_{1}\right\} .
$$

Thus $p \geq m_{1}=\operatorname{dim} \operatorname{ker} A$. It is now sufficient to prove

$$
\operatorname{ker} A^{2}=\operatorname{ker} A \text {, }
$$

since this implies ker $A^{i}=\operatorname{ker} A$ for all $i \geq 1$, yielding immediately that $p=m_{1}$.

It is known that (6.5) holds if and only if ker $A \cap \operatorname{Range}(A)=\{0\}$. Let $A^{*}$ be the adjoint operator of $A$, then Range $(A)=\left(\operatorname{ker} A^{*}\right)^{\perp}$. Let $(\phi, \psi) \in \operatorname{ker} A^{*}$. Then $K^{*}(\phi, \psi)=\left(\mu_{1}+1\right)(\phi, \psi)$, where $K^{*}$ is the adjoint of $K$. This gives

$$
2 f_{0} G(\phi)+g_{0} G_{d}(\psi)=\left(\mu_{1}+1\right) \phi
$$

and

$$
f_{1} G(\phi)=\left(\mu_{1}+1\right) \psi .
$$

By the definition of $G$ and $G_{d}$ we obtain

$$
\begin{aligned}
-d f_{1}\left(\mu_{1}+1\right) \Delta \phi & =f_{\phi} \phi+f_{\psi} \psi, \\
-\left(\mu_{1}+1\right) \Delta \psi & =f_{1} \phi-\left(\mu_{1}+1\right) f_{0} \psi,
\end{aligned}
$$

where

$$
f_{\phi}=2 d f_{0} f_{1}+\left(\mu_{1}+1\right) f_{1} g_{1}, \quad f_{\psi}=f_{1} g_{0}-2\left(\mu_{1}+1\right)\left(f_{0} g_{1}+d f_{0}^{2}\right) .
$$

Using the same Fourier expansion for $\phi$ and $\psi$ as above we have $B_{i}^{*}\left(\begin{array}{l}a_{i j} \\ b_{i j}\end{array}\right)=0$, where $0 \leq i \leq \infty, 1 \leq j \leq m_{i}$, and

$$
B_{i}^{*}=\left(\begin{array}{cc}
f_{\phi}-d f_{1}\left(\mu_{1}+1\right) \lambda_{i} & f_{\psi} \\
f_{1} & -\left(\mu_{1}+1\right)\left(f_{0}+\lambda_{i}\right)
\end{array}\right) .
$$

If we replace $\mu$ by $\mu_{1}$ in the matrix $B_{i}$, then it can be verified that $\operatorname{det} B_{i}^{*}=f_{1} \operatorname{det} B_{i}$. Hence $\operatorname{det} B_{1}^{*}=0$, and $\operatorname{det} B_{i}^{*} \neq 0$ for $i \geq 2$. It follows that

$$
\operatorname{ker} A^{*}=\left\{\left(\begin{array}{c}
\left(\mu_{1}+1\right)\left(\lambda_{1}+f_{0}\right) \\
f_{1}
\end{array}\right) \phi_{1 j}, 1 \leq j \leq m_{1}\right\} .
$$

Since

$$
\left(\mu_{1}-1\right) f_{0}+\left(\mu_{1}+1\right) \lambda_{1}+\left(\mu_{1}+1\right)\left(\lambda_{1}+f_{0}\right)=2\left(\mu_{1}+1\right) \lambda_{1}+2 \mu_{1} f_{0}>0,
$$

we conclude that ker $A \cap\left(\operatorname{ker} A^{*}\right)^{\perp}=\{0\}$, which leads to $p=m_{1}$ and a contradiction to (6.2). This completes the proof. 
One can easily extend the argument in the proof of this theorem to obtain a more general result without assuming $f_{0}<\lambda_{2}$, but the statement can be complicated since $d_{i}$, defined in Section 5 , is not monotone as a function of $i$. We shall leave the details to the interested readers.

\section{REFERENCES}

[CK] T. K. Callahan and E. Knobloch, Pattern formation in three-dimensional reactiondiffusion systems, Phys. D 132, 339-362 (1999). MR1701288 (2000e:35098)

[CH] R. G. Casten and C. J. Holland, Stability properties of solutions to systems of reaction-diffusion equations, SIAM J. Appl. Math. 33, 353-364 (1977). MR447760 (56 \#6070)

[CDBD] V. Castets, E. Dulos, J. Boissonade and P. De Kepper, Experimental evidence of a sustained Turing-type equilibrium chemical pattern, Phys. Rev. Lett. 64, 2953-2956 (1990).

[DCDB] P. De Kepper, V. Castets, E. Dulos and J. Boissonade, Turing-type chemical patterns in the chlorite-iodide-malonic acid reaction, Physica D 49, 161-169 (1991).

[F] A. Friedman, Partial Differential Equations of Parabolic Type. Prentice-Hall: Englewood Cliffs, NJ, 1964. MR0181836 (31 \#6062)

[GM] A. Gierer and H. Meinhardt, A theory of biological pattern formation, Kybernetik 12, 30-39 (1972).

[JNT] J. JANG, W.-M. Ni AND M. TANG, Global bifurcation and structure of Turing patterns in 1-D Lengyel-Epstein model, J. Dynam. Diff. Eqs. 16 297-320 (2004). MR2105777

[JMBD] O. Jensen, E. Mosekilde, P. Borckmans and G. Dewel, Computer simulations of Turing structures in the Chlorite-Iodide-Malonic Acid system, Physica Scripta 53, 243251 (1996).

[JS] S. L. Judd And M. Silber, Simple and superlattice Turing patterns in reaction-diffusion systems: bifurcation, bistability, and parameter collapse, Phys. D 136, 45-65 (2000). MR.1732305 (2001a:37125)

[LE1] I. LEnGYel AND I. R. Epstein, Modeling of Turing structures in the chlorite-iodidemalonic acid-starch reaction system, Science 251, 650-652 (1991).

[LE2] I. Lengyel And I. R. Epstein, A chemical approach to designing Turing patterns in reaction-diffusion systems, Proc. Natl. Acad. Sci. USA 89, 3977-3979 (1992).

[LN] Y. Lou AND W.-M. Ni, Diffusion, self-diffusion and cross-diffusion, J. Differential Equations 131, 79-131 (1996). MR1415047 (97i:35086)

[N] W.-M. NI, Diffusion, cross-diffusion, and their spike-layer steady states, Notices Amer. Math. Soc. 45, 9-18 (1998). MR 1490535 (99a:35132)

[Tr] A. Trembley, Memoires pour servir a l'histoire d'un genre de polypes d'eau douce a bras en forme de cornes, Leyden, 1744.

[T] A. M. Turing, The chemical basis of morphogenesis, Phil. Trans. Roy. Soc. London Ser. B 237, 37-72 (1952).

[WS] D. J. Wollkind and L. E. Stephenson, Chemical Turing pattern formation analyses: comparison of theory with experiment, SIAM J. Appl. Math. 61, 387-431 (2000). MR:1780797 (2001g:76062)

[W] H. F. WEINBERGER, Invariant sets for weakly coupled parabolic and elliptic systems, Rend. Mat. 8, 295-310 (1975). MR0397126|(53 \#986)

School of Mathematics, University of Minnesota, Minneapolis, Minnesota 55455

E-mail address: ni@math.umn.edu

Department of Mathematics, Michigan State University, East Lansing, Michigan 48824

E-mail address: mtang@math.msu.edu 